\title{
EFFECT OF INDUCED MAGNETIC FIELD ON MHD MIXED CONVECTION FLOW IN VERTICAL MICROCHANNEL
}

\author{
B.K. JHA and B. AINA* \\ Department of Mathematics, Ahmadu Bello University \\ Zaria, NIGERIA \\ E-mails: basant777@yahoo.co.uk; ainavicdydx@gmail.pl
}

\begin{abstract}
The present work presents a theoretical investigation of an MHD mixed convection flow in a vertical microchannel formed by two electrically non-conducting infinite vertical parallel plates. The influence of an induced magnetic field arising due to motion of an electrically conducting fluid is taken into consideration. The governing equations of the motion are a set of simultaneous ordinary differential equations and their exact solutions in dimensionless form have been obtained for the velocity field, the induced magnetic field and the temperature field. The expressions for the induced current density and skin friction have also been obtained. The effects of various non-dimensional parameters such as rarefaction, fluid wall interaction, the Hartmann number and the magnetic Prandtl number on the velocity, the induced magnetic field, the temperature, the induced current density, and skin friction have been presented in a graphical form. It is found that the effect of the Hartmann number and magnetic Prandtl number on the induced current density is found to have a decreasing nature at the central region of the microchannel.
\end{abstract}

Key words: microchannel, transverse magnetic field, induced magnetic field, velocity slip, temperature jump.

\section{Introduction}

Studies associated with a mixed convection flow of an electrically conducting fluid in the presence of an external magnetic field have received considerable interest due to the enormous applications in various branches of industry, science and technology such as fire engineering, combustion modelling, geophysics, the cooling of nuclear reactors, operation of magnetohydrodynamic (MHD) generators, plasma studies, etc. Application of a magnetic field has been found to be effective in controlling the melt convection during crystal growth from melts under terrestrial conditions and has now been widely used in metal and semiconductor industries. Several studies have been reported on an MHD convective flow under different physical situations. Record of such investigations can be found in the works of Cramer and Pai [1], Chawla [2], Das et al. [3], Sheikholesslami and Gorgi-Bandpy [4], Sheikholesslami et al. [5], Sheikholesslami et al. [6], Chauhan and Rastogi [7], Ibrahim and Makinde. [8], Farhad et al. [9-10].

Although there are many studies on natural convection flow of an electrically conducting fluid in channels, there are only a few studies regarding natural and mixed convection flow of an electrically conducting fluid in a microchannel and annular microchannel. In recent years, the present authors and their collaborators have carried out a number of studies on MHD natural convection covering several aspects. For instance, Jha et al. [11] analytically studied the fully developed steady natural convection flow of a conducting fluid in a vertical parallel plate microchannel in the presence of a transverse magnetic field. The effect of the Hartmann number was reported to decrease the volume flow rate. The combined influence of an externally applied transverse magnetic field and suction/injection on a steady natural convection flow of conducting fluid in a vertical microchannel was carried out by Jha et al. [12]. In another work, Jha et al. [13] examined the effect of wall surface curvature on the transient MHD free convective flow in a vertical micro-

\footnotetext{
* To whom correspondence should be addressed
} 
concentric-annuli. Jha et al. [14] studied exact solution of a steady fully developed natural convection flow of a viscous, incompressible, and electrically conducting fluid in a vertical annular microchannel. Rececntly, Jha and Aina [15] presented the MHD natural convection flow in a vertical micro-porous-annulus (MPA) in the presence of radial magnetic field. Also, the MHD natural convection flow in vertical micro-concentricannuli (MCA) in the presence of radial magnetic field has been analyzed by Jha et al. [16].

The above studies on MHD natural convective heat and mass transfer in a vertical microchannel and annular microchannel have been limited to the cases in which the induced magnetic field is neglected in order to facilitate the mathematical analysis of the problem. However, the induced magnetic field also generates its own magnetic field in the fluid and as a result it modifies the applied magnetic field and motion of the fluid. Therefore, it is known that in several physical situations, it will be necessary to include the effect of the induced magnetic field in the MHD equations when the magnetic Reynolds number is large enough [1]. Singh et al. [17] presented numerical studies on the hydromagnetic free convective flow in the presence of induced magnetic field. Jha and Sani [18] presented the MHD natural convection flow of an electrically conducting and viscous incompressible fluid in a vertical channel due to symmetric heating in the presence of an induced magnetic field. A study on hydromagnetic free convective flow in the presence of an induced magnetic field was carried out by Ghosh et al. [19]. In another related work, Kumar and Singh [20] studied the unsteady MHD free convective flow past a semi- infinite vertical wall by taking into account the induced magnetic field. Recently, Sarveshanand and Singh [21] analytically studied the MHD free convective flow between vertical parallel porous plates in the presence of an induced magnetic field and found that the induced current density profile increases with an increase in the magnetic Prandtl number.

The induced magnetic field has many important applications in the experimental and theoretical studies of the MHD flow due to its use in many scientific and technological phenomena, for example in MHD electrical power generation, geophysics, purification of crude oil, glass manufacturing, etc. The role of the induced magnetic field is important when the magnetic Reynolds number is large enough [1].

The objective of this work is to present a comprehensive theoretical study of a steady hydromagnetic fully developed mixed convection flow in a vertical microchannel formed by two infinite vertical parallel plates in the presence of an induced magnetic field. Both walls of the microchannel are electrically nonconducting and maintained at different temperatures.

\section{Mathematical analysis}

A steady laminar fully developed mixed convection flow of an electrically conducting, viscous incompressible fluid in a vertical microchannel formed by two electrically non-conducting infinite vertical parallel plates is considered. The $x^{\prime}$-axis is taken vertically upward along the plates and the $y^{\prime}-$ axis normal to it as presented in Fig.1. A magnetic field of uniform strength $H_{0}^{\prime}$ is assumed to be applied in the direction perpendicular to the direction of flow. Both plates $y^{\prime}=0$ and $y^{\prime}=b$ are taken to be nonconducting. As stated earlier, our emphasis in this study is to investigate the effect of an induced magnetic field on the flow formation inside the vertical microchannel. It is shown that the fluid flow and heat transfer at microscale differ greatly from those at macroscale. At macroscale, classical conservation equations are successfully coupled with the corresponding wall boundary conditions which are valid only if the fluid flow adjacent to the surface is in thermal equilibrium. However, they are not valid for the fluid flow at microscale. For this case, the fluid no longer reaches the velocity or the temperature of the surface and therefore a slip condition for the velocity and a jump condition for the temperature should be adopted. In the present study, the usual continuum approach is applied by the continuum equations with the two main characteristics of the microscale phenomena, the velocity slip and the temperature jump. The velocity slip is defined as [22]

$$
u_{s}=-\left.\frac{2-\sigma_{v}}{\sigma_{v}} \lambda \frac{\partial u}{\partial y}\right|_{y=b}
$$


where $u_{s}$ is the slip velocity, $\lambda$ is the molecular mean free path and $\sigma_{v}$ is the tangential momentum accommodation coefficient, and the temperature jump is defined as [22]

$$
T_{s}-T_{w}=-\left.\frac{2-\sigma_{t}}{\sigma_{t}} \frac{2 \gamma}{\gamma+1} \frac{\lambda}{\operatorname{Pr}} \frac{\partial T}{\partial y}\right|_{y=b}
$$

where $T_{s}$ is the temperature of the fluid at the wall, $T_{w}$ is the wall temperature, and $\sigma_{t}$ is the thermal accommodation coefficient, which depends on the gas and surface materials. However, for air, it assumes typical values near unity [22]. For the rest of the analysis, $\sigma_{v}$ and $\sigma_{t}$ will be assumed to be 1.

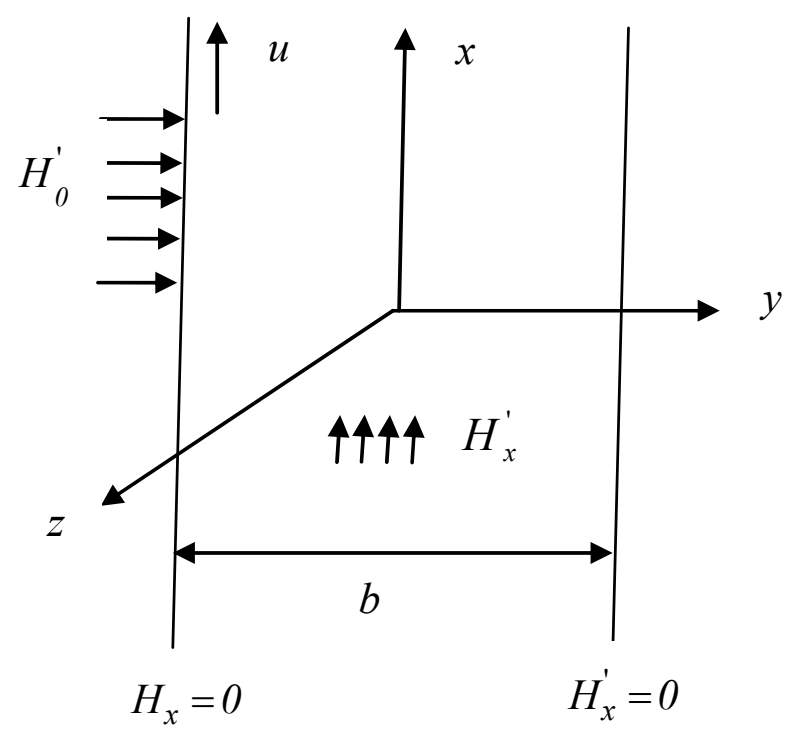

Fig.1. Flow configuration and coordination.

In a sufficiently long channel, the velocity and temperature profiles will cease to change with distance along the channel, i.e., a fully developed flow will exist [23]. Assuming a hydrodynamically fully developed flow, the transverse velocity $v$ becomes equal to zero. Then, the continuity equation drops to $\partial u / \partial x=0$. Further, the viscous dissipation and compressibility effects in the fluid are neglected in order to derive the exact solution of the present physical situation. By taking into account the conducting fluid, transverse magnetic field and induced magnetic field and substituting the above conditions into the governing equations of the heat and fluid flow, we obtain the dimensional form

$$
\begin{aligned}
& \cup \frac{d^{2} u^{\prime}}{d y^{\prime 2}}+\frac{\mu_{e} H_{0}^{\prime}}{\rho} \frac{d H_{x}^{\prime}}{d y^{\prime}}-\frac{d p}{d x}+g \beta\left(T^{\prime}-T_{0}\right)=0, \\
& \frac{1}{\sigma \mu_{e}} \frac{d^{2} H_{x}^{\prime}}{d y^{\prime 2}}+H_{0}^{\prime} \frac{d u^{\prime}}{d y^{\prime}}=0, \\
& \frac{d^{2} T^{\prime}}{d y^{\prime 2}}=0
\end{aligned}
$$

with the boundary conditions for the velocity, induced magnetic field and temperature field which are 


$$
\begin{aligned}
& u^{\prime}(y=0)=\frac{2-\sigma_{v}}{\sigma_{v}} \lambda \frac{d u^{\prime}}{d y^{\prime}}, \quad H_{x^{\prime}}^{\prime}=0, \quad T^{\prime}\left(y^{\prime}=0\right)=T_{2}+\frac{2-\sigma_{t}}{\sigma_{t}} \frac{2 \gamma}{\gamma+1} \frac{\lambda}{\operatorname{Pr}} \frac{d T^{\prime}}{d y^{\prime}} \quad \text { at } \quad y^{\prime}=0, \\
& u^{\prime}\left(y^{\prime}=b\right)=-\frac{2-\sigma_{v}}{\sigma_{v}} \lambda \frac{d u^{\prime}}{d y^{\prime}}, H_{x^{\prime}}^{\prime}=0, \quad T^{\prime}\left(y^{\prime}=b\right)=T_{1}-\frac{2-\sigma_{t}}{\sigma_{t}} \frac{2 \gamma}{\gamma+1} \frac{\lambda}{\operatorname{Pr}} \frac{d T^{\prime}}{d y^{\prime}} \text { at } y^{\prime}=b .
\end{aligned}
$$

In the above Eqs $(2.3)-(2.7), T^{\prime}$ is the temperature of the fluid, $\boldsymbol{H}\left[=\left(H_{x}^{\prime}, H_{0}^{\prime}, 0\right)\right]$ is the magnetic field, $v$ is the kinematic viscosity, $g$ is the acceleration due to gravity, $\beta$ is the coefficient of thermal expansion, $\mu_{e}$ is the magnetic permeability, $\rho$ is the density, and $\sigma$ is the electrical conductivity of the fluid.

Using the following non-dimensional quantities

$$
\begin{aligned}
& y=\frac{y^{\prime}}{b}, \quad U_{0}=\frac{v u^{\prime}}{g \beta b^{2}\left(T_{1}-T_{0}\right)}, \quad \theta=\frac{T^{\prime}-T_{0}}{T_{1}-T_{0}}, \quad \operatorname{Pm}=v \sigma \mu_{e}, \quad \operatorname{Gr}=\frac{g \beta\left(T_{1}-T_{0}\right) b^{3}}{v^{2}}, \\
& \operatorname{Pr}=\frac{v}{\alpha}, \quad \operatorname{Re}=\frac{U_{0} b}{v}, \quad H=\frac{v H_{x^{\prime}}^{\prime}}{g \beta b^{2}\left(T_{1}-T_{0}\right)} \sqrt{\frac{\mu_{e}}{\rho}}, \quad \mathrm{M}=\frac{H_{0} b}{v} \sqrt{\frac{\mu_{e}}{\rho}},
\end{aligned}
$$

the governing equations in a non-dimensional form have taken the form

$$
\begin{aligned}
& \frac{d^{2} U}{d y^{2}}+M \frac{d H}{d y}+\frac{\mathrm{Gr}}{\operatorname{Re}} \theta-\frac{d p}{d X}=0, \\
& \frac{d^{2} H}{d y^{2}}+M \operatorname{Pm} \frac{d U}{d y}=0, \\
& \frac{d^{2} \theta}{d y^{2}}=0,
\end{aligned}
$$

with the boundary conditions in a non dimensional form as

$$
\begin{array}{ll}
U(y)=\beta_{v} \operatorname{Kn} \frac{d U}{d y}, \quad H=0, \quad \theta(y)=\xi+\beta_{v} \operatorname{Kn} \ln \frac{d \theta}{d y} \quad \text { at } \quad y=0, \\
U(y)=-\beta_{v} \operatorname{Kn} \frac{d U}{d y}, \quad H=0, \quad \theta(y)=1-\beta_{v} \operatorname{Kn} \ln \frac{d \theta}{d y} \quad \text { at } \quad y=1
\end{array}
$$

where

$$
\beta_{v}=\frac{2-\sigma_{v}}{\sigma_{v}}, \quad \beta_{t}=\frac{2-\sigma_{t}}{\sigma_{t}} \frac{2 \gamma_{s}}{\gamma_{s}+1} \frac{1}{\operatorname{Pr}}, \quad K n=\frac{\lambda}{b}, \quad \ln =\frac{\beta_{t}}{\beta_{v}}, \quad \xi=\frac{T_{2}-T_{0}}{T_{1}-T_{0}} .
$$

Referring to the values of $\sigma_{v}$ and $\sigma_{t}$ given in Eckert and Drake [24] and Goniak and Duffa [25], the value of $\beta_{v}$ is near unity, and the value of $\beta_{t}$ ranges from near 1 to more than 100 for actual wall surface 
conditions and is near 1.667 for many engineering applications, corresponding to $\sigma_{v}=1, \sigma_{t}=1, \gamma_{s}=1.4$ and $\operatorname{Pr}=0.71\left(\beta_{v}=1, \beta_{t}=1.667\right)$.

The physical quantities used in the above equations are defined in the nomenclature.

\section{Method of solution}

Equations (2.9), (2.10), (2.11) are a coupled system of ordinary differential equations with constant coefficients. This system of linear ordinary differential equations has been solved in closed form by the theory of simultaneous ordinary differential equations. The expressions for the velocity field, the induced magnetic field and the temperature field in non-dimensional form are given by

$$
\begin{aligned}
& U(y)=C_{4} \cosh (y M \sqrt{\mathrm{Pm}})+C_{5} \sinh (y M \sqrt{\mathrm{Pm}})+\frac{\mathrm{Gr} A_{1} y}{\mathrm{Re}}-C_{1} A_{2}, \\
& H(y)=\frac{C_{6}}{M}-\frac{\mathrm{Gr} A_{1}}{\operatorname{Re} M}-\frac{\mathrm{Gr}}{\mathrm{ReM}}\left[0.5 C_{1} y^{2}+C_{2} y\right]+\frac{d P}{d x} \frac{y}{M}+ \\
& -\sqrt{\mathrm{Pm}}\left[C_{4} \sinh (y M \sqrt{\mathrm{Pm}})+C_{5} \cosh (y M \sqrt{\mathrm{Pm}})\right], \\
& \theta(y)=C_{1} y+C_{2} .
\end{aligned}
$$

The induced current density is given by

$$
\begin{aligned}
& J=-\frac{d H}{d y} \\
& =\frac{\mathrm{Gr}}{\operatorname{Re} M}\left[C_{1} y+C_{2}\right]-\frac{d P}{d X} \frac{1}{M}+M \operatorname{Pm}\left[C_{4} \cosh (y M \sqrt{\mathrm{Pm}})+C_{5} \sinh (y M \sqrt{\mathrm{Pm}})\right]
\end{aligned}
$$

where $C_{1}, \ldots, C_{6}$ are all constants given in the Appendix.

Two important parameters for buoyancy - induced micro-flow are the volume flow rate $\left(Q_{m}\right)$ and skin friction $(\tau)$. The dimensionless volume flow rate is

$$
\begin{aligned}
& Q_{m}=\int_{0}^{1} U(y) d y, \\
& =\frac{1}{M \sqrt{\mathrm{Pm}}}\left[C_{4} \sinh (M \sqrt{\mathrm{Pm}})+C_{5}\{\cosh (M \sqrt{\mathrm{Pm}})-1\}\right]+\frac{\mathrm{Gr} A_{1}}{2 \mathrm{Re}}-A_{2} C_{3} .
\end{aligned}
$$

Using expression (3.1), the skin - friction on both microchannel walls in a dimensionless form are given by

$$
\tau_{0}=\left.\frac{d U}{d y}\right|_{y=0},
$$




$$
\begin{aligned}
& =M \sqrt{\mathrm{Pm}} C_{5}+\frac{\mathrm{Gr} A_{1}}{\mathrm{Re}}, \\
& \tau_{0}=\left.\frac{d U}{d y}\right|_{y=0}, \\
& =M \sqrt{\mathrm{Pm}}\left[C_{4} \sinh (M \sqrt{\mathrm{Pm}})+C_{5} \cosh (M \sqrt{\mathrm{Pm}})\right]+\frac{\mathrm{Gr} A_{1}}{\operatorname{Re}} .
\end{aligned}
$$

\section{Results and discussion}

The present study on a magnetohydrodynamic mixed convection flow in a vertical microchannel formed by two electrically non-conducting infinite vertical parallel plates in the presence of an induced magnetic field is controlled by a number of physical parameters such as the mixed convection parameter $(\mathrm{Gr} / \mathrm{Re})$, wall-ambient temperature difference ratio $(\xi)$, rarefaction $\left(\beta_{v} K n\right)$, fluid wall interaction $(\ln )$, the Hartmann number (M), and the magnetic Prandtl number (Pm). The effects of these parameters on the velocity profile, induced magnetic field profile, induced current density profile and the skin friction are shown using the line graphs.

The expression for the temperature in Eq.(3.3), the effects of the rarefaction parameter $\left(\beta_{v} K n\right)$, and fluid wall interaction parameter $(\ln )$ on the temperature profile and rate of heat transfer which is expressed as the Nusselt number are exactly the same as those given by Chen and Weng [26].

Figure 2 exhibit the effect of the mixed convection parameter $(\mathrm{Gr} / \mathrm{Re})$ and wall-ambient temperature difference ratio $(\xi)$, on the velocity profile for a fixed value of $\beta_{v} K n=0.05, \ln =1.667, \mathrm{M}=5.0$, and $\mathrm{Pm}=0.5$. It is found that near the channel wall $(y=0)$, increasing the mixed convection parameter $(\mathrm{Gr} / \mathrm{Re})$ leads to a reduction in fluid velocity while the result is just converse at the channel wall $(y=1)$. Further, at the middle of the channel, the fluid flow is not affected by the mixed convection parameter $(\mathrm{Gr} / \mathrm{Re})$. Physically speaking, increasing $(\mathrm{Gr} / \mathrm{Re}>0)$ means convection current and pressure gradient support each other, hence, an increase in the fluid velocity while $(\mathrm{Gr} / \mathrm{Re}<0)$ shows natural convection opposing the effect of the pressure gradient. Furthermore, for large values of the mixed convection parameter $(\mathrm{Gr} / \mathrm{Re})$ there exist reversed flow regions with negative velocity values at channel wall $(y=0)$. Also, an increase in the mixed convection parameter $(\mathrm{Gr} / \mathrm{Re})$ increases this reversed flow region and the critical value of the mixed convection parameter $(\mathrm{Gr} / \mathrm{Re})$ leading to the flow reversal is $\left.\frac{\mathrm{Gr}}{\mathrm{Re}}\right|_{\text {critical }}=110$. In addition, the flow reversal is possible at the channel wall $(y=1)$ by considering negative values of mixed convection parameter.

Figure 3 illustrates the effects of the rarefaction parameter $\left(\beta_{v} K n\right)$, and wall-ambient temperature difference ratio $(\xi)$, on velocity profiles for fixed values of $\mathrm{Gr} / \mathrm{Re}=100, \ln =1.667, \mathrm{M}=5.0$, and $\mathrm{Pm}=0.5$. It is noticed that an increase in the rarefaction parameter and wall-ambient temperature difference ratio causes a pronounced enhancement in the velocity slip. This result yields an observable increase in the fluid velocity. This effect can be explained by the fact that as the rarefaction parameter increases, the temperature jump increases and this reduces the amount of heat transfer from the microchannel surfaces to the fluid. The reduction in velocity due to the reduction in heat transfer is offset by the increase in the fluid velocity due to the reduction in the frictional retarding forces near the microchannel surfaces. 
Furthermore, as the wall-ambient temperature difference ratio $(\xi)$, increases, the effect of the rarefaction parameter $\left(\beta_{v} K n\right)$, on the microchannel slip velocity becomes significant.

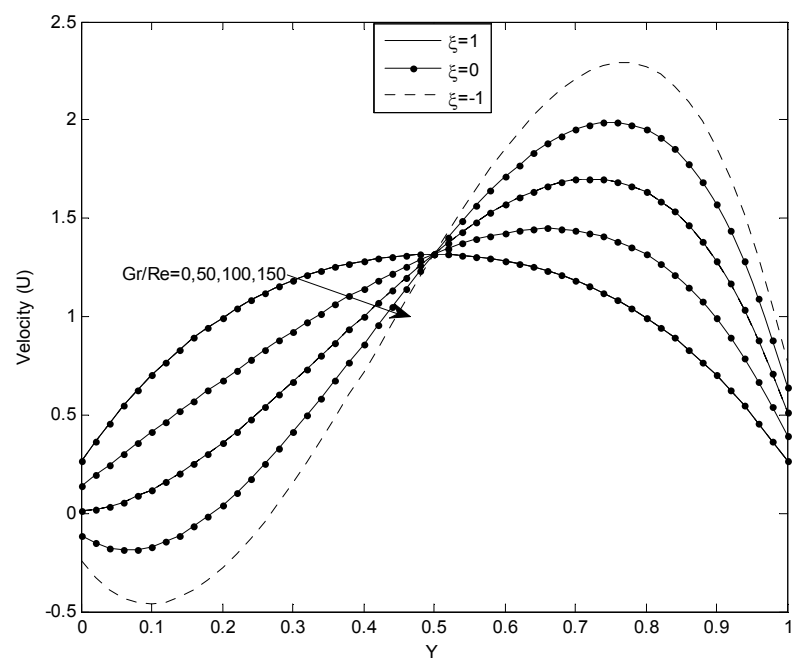

Fig.2. Variation of velocity with $\mathrm{Gr} / \mathrm{Re}(\mathrm{M}=5.0$, $\left.\mathrm{Pm}=0.5, \beta_{v} \mathrm{Kn}=0.05, \mathrm{In}=1.667\right)$.

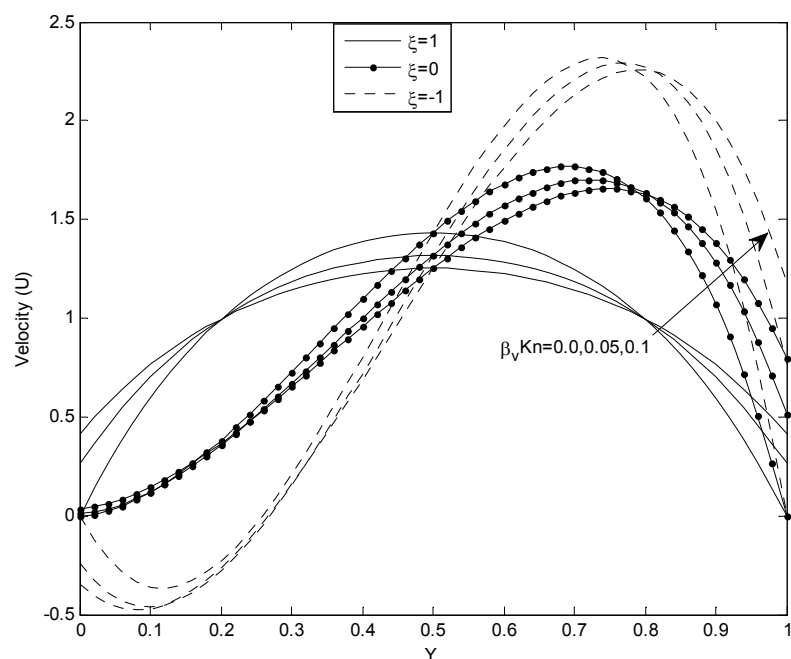

Fig.3. Variation of velocity with $\beta_{v} \mathrm{Kn} \quad(\mathrm{M}=5.0$, $\mathrm{Pm}=0.5, \mathrm{Gr} / \mathrm{Re}=100, \mathrm{In}=1.667)$.

Figure 4 illustrate the effects of the fluid wall interaction parameter $(\ln )$ as well as wall-ambient temperature difference ratio $(\xi)$, on velocity profiles for fixed values of $\mathrm{Gr} / \mathrm{Re}=100, \beta_{v} K n=0.05$, $\mathrm{M}=5.0$, and $\mathrm{Pm}=0.5$. It is observed that the effect of the fluid wall interaction parameter is to enhance the fluid velocity at the microchannel wall $(y=0)$ and to reduce the fluid velocity at the microchannel wall $(y=1)$. In addition, it is evident that there exist points of intersection inside the microchannel where the velocity profile is independent of the fluid wall interaction parameter. Also, the impacts of the fluid-wall interaction parameter on the microchannel slip velocity become significant with the decrease of the wallambient temperature difference ratio.

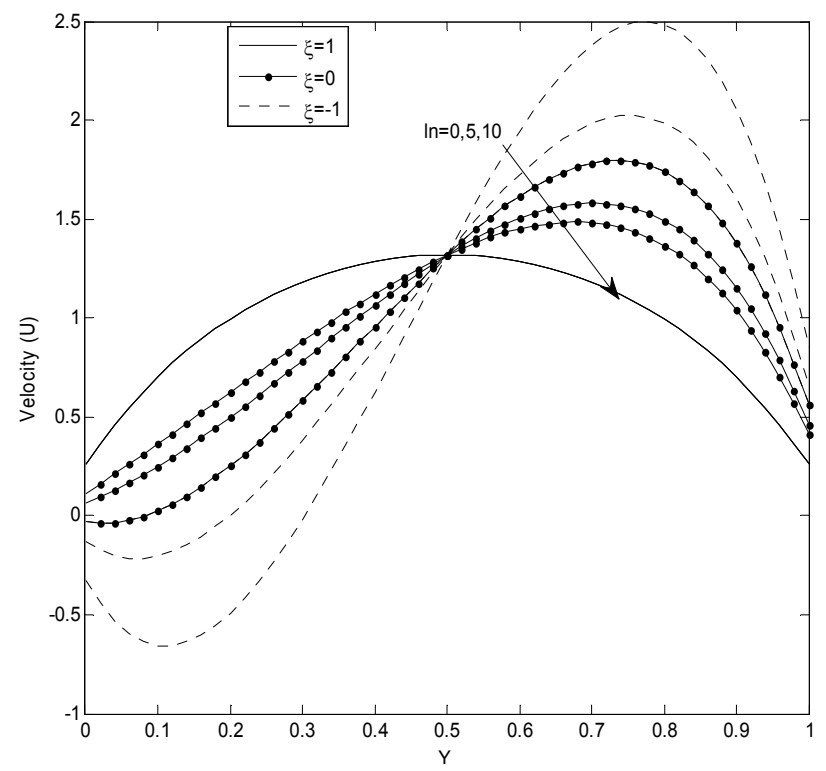

Fig.4. Variation of velocity with $\ln \left(\mathrm{M}=5.0, \mathrm{Pm}=0.5, \mathrm{Gr} / \mathrm{Re}=100, \beta_{v} \mathrm{Kn}=0.05\right)$. 
Figures 5 and 6 exhibit the effects of the wall-ambient temperature difference ratio $(\xi)$, the Hartmann number $(\mathrm{M})$, and the magnetic Prandtl number $(\mathrm{Pm})$, respectively, on velocity profiles for fixed values of $\mathrm{Gr} / \mathrm{Re}=100, \beta_{v} K n=0.05$, and $\ln =1.667$. It is evident from these figures that an increase in the Hartmann number and the magnetic Prandtl number causes a reduction in the fluid velocity at the microchannel wall $(y=1)$. Physically speaking, the presence of a transverse magnetic field sets in a resistive type force (Lorentz force), which is a retarding force on the velocity field. It is further observed that there exist points of intersection inside the microchannel where the velocity field is independent of the Hartmann number and the magnetic Prandtl number and this behaviour is observed in the case of asymmetric heating $(\xi=-1)$. Also, the influence of the Hartmann number and the magnetic Prandtl number on the microchannel slip velocity becomes significant with the increase of the wall-ambient temperature difference ratio.

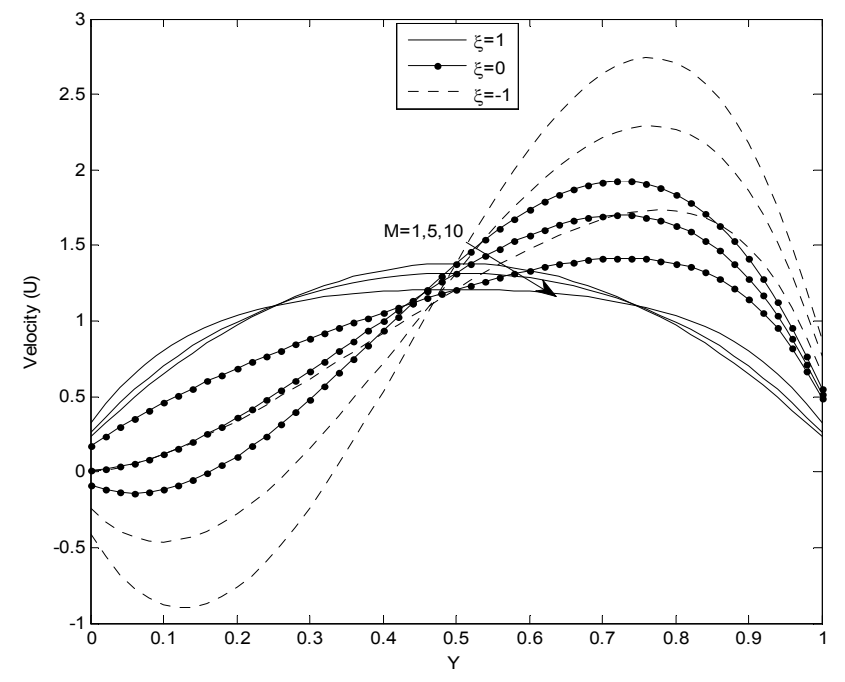

Fig.5. Variation of velocity with $\mathrm{M}(\mathrm{In}=1.667$, $\mathrm{Pm}=0.5, \mathrm{Gr} / \mathrm{Re}=100, \beta_{v} \mathrm{Kn}=0.05$ ).

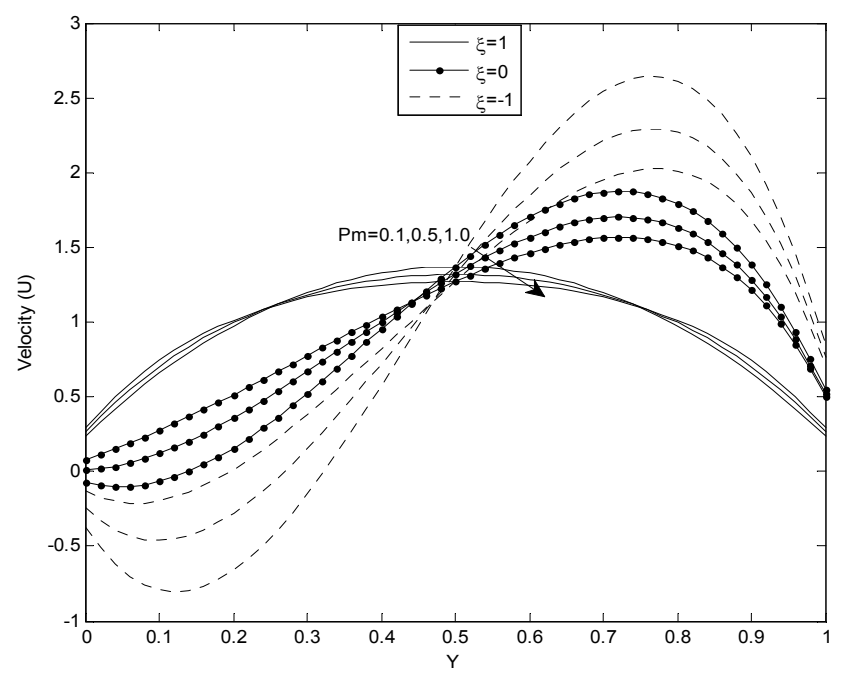

Fig.6. Variation of velocity with $\mathrm{Pm}(\mathrm{In}=1.667$, $\left.\mathrm{M}=5, \mathrm{Gr} / \mathrm{Re}=100, \beta_{v} \mathrm{Kn}=0.05\right)$.

Figure 7 shows the induced magnetic field with respect to the mixed convection parameter $(\mathrm{Gr} / \mathrm{Re})$ on the velocity profile for a fixed value of $\ln =1.667, \beta_{v} K n=0.05, \mathrm{M}=5.0$, and $\mathrm{Pm}=0.5$. It is evident from Fig. 8 that an increase in the mixed convection parameter causes a pronounced enhancement in the induced magnetic field.

Figure 8 depicts the distribution of the induced magnetic field with respect to the rarefaction parameter $\left(\beta_{v} K n\right)$, and wall-ambient temperature difference ratio $(\xi)$. It is observed that, the rarefaction parameter influences the flow formation excluding the case of symmetric heating $(\xi=1)$. For the case of asymmetric heating $(\xi=0,-1)$, it is found that the induced magnetic field increases with the increase in the rarefaction parameter. It is further noticed from Figure 6 that the role of the rarefaction parameter on the induced magnetic field is more pronounced with a reduction in the values of the wall-ambient temperature difference ratio. 


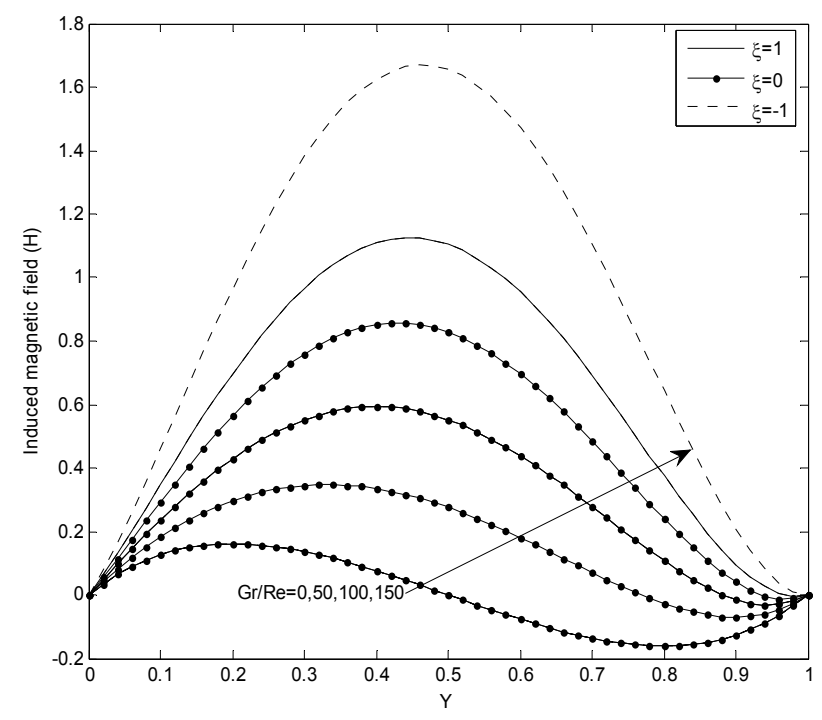

Fig.7. Variation of induced magnetic field with $\mathrm{Gr} / \mathrm{Re}$ $\left(\mathrm{M}=5.0, \mathrm{Pm}=0.5, \beta_{v} \mathrm{Kn}=0.05, \mathrm{In}=1.667\right)$.

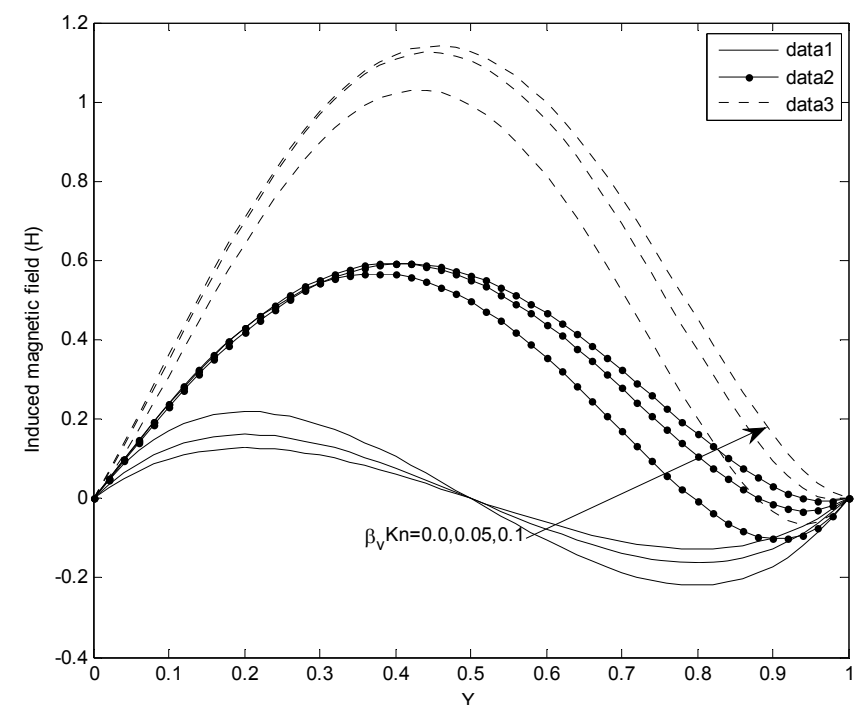

Fig.8. Variation of induced magnetic field with $\beta_{v} \mathrm{Kn}$ $(\mathrm{M}=5.0, \mathrm{Pm}=0.5, \mathrm{Gr} / \mathrm{Re}=100, \mathrm{In}=1.667)$.

Figure 9 shows the induced magnetic field with respect to the fluid wall interaction parameter $(\ln )$ and wall-ambient temperature difference ratio $(\xi)$. It is evident from Fig.8 that an increase in the fluid wall interaction parameter causes a pronounced reduction in the fluid velocity. Also, the impacts of the fluid-wall interaction parameter on the induced magnetic field become significant with the decrease in the wall-ambient temperature difference ratio.

The induced magnetic field profile is plotted in Figs 10 and 11 with various values of the Hartmann number (M) and the magnetic Prandtl number $(\mathrm{Pm})$, respectively. It is interesting to note that the strength of the induced magnetic field is directly proportional to the strength of the Hartmann number as well as the magnetic Prandtl number near the channel wall at $(y=0)$ while it is inversely proportional near the channel wall at $(y=1)$. Furthermore, there exist points of intersection inside the vertical channel where the induced magnetic field is independent of the Hartmann number and the magnetic Prandtl number.

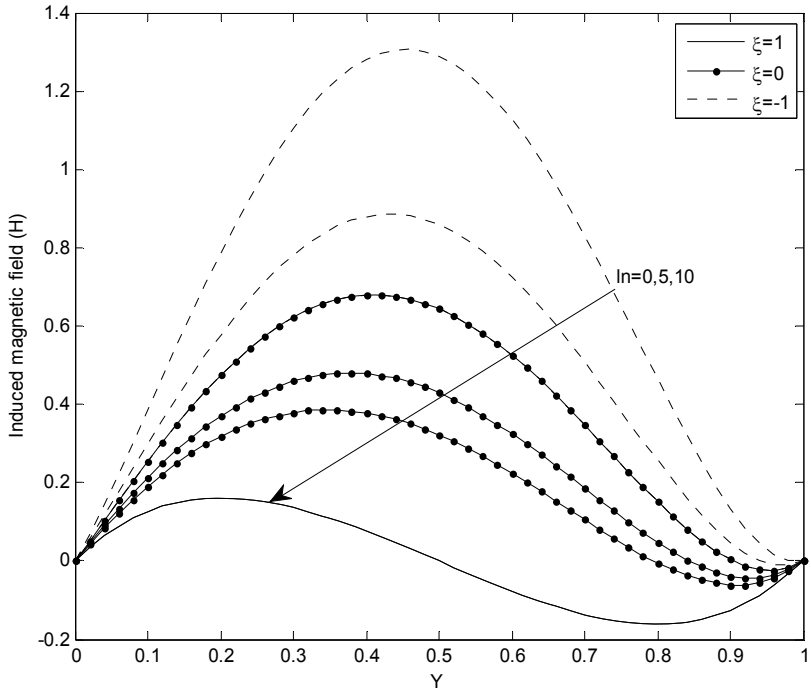

Fig.9. Variation of induced magnetic field with In $\left(\mathrm{M}=5.0, \mathrm{Pm}=0.5, \mathrm{Gr} / \mathrm{Re}=100, \beta_{v} \mathrm{Kn}=0.05\right)$.

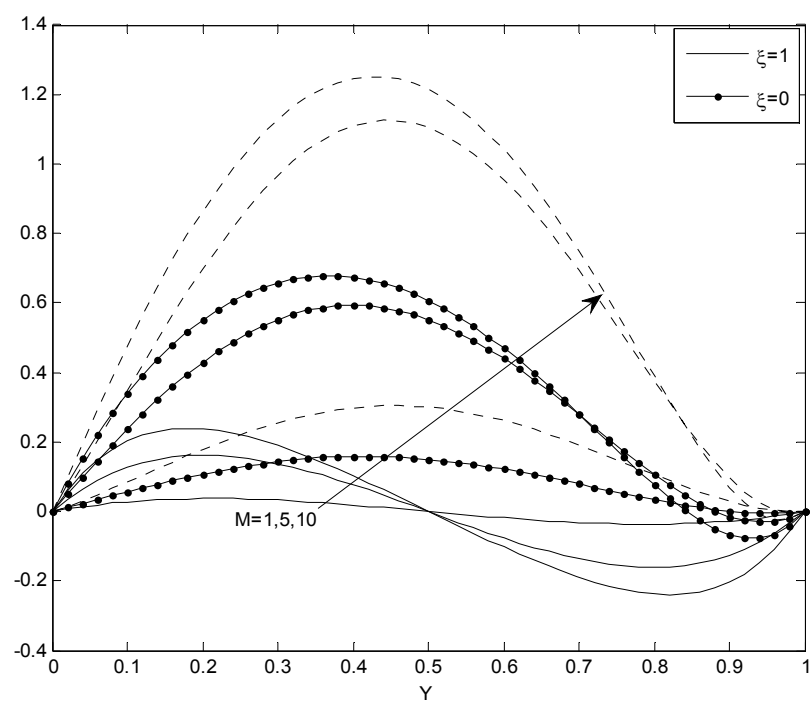

Fig.10. Variation of induced magnetic field with $\mathrm{M}$ $\left(\mathrm{In}=1.667, \mathrm{Pm}=0.5, \mathrm{Gr} / \mathrm{Re}=100, \beta_{v} \mathrm{Kn}=0.05\right)$. 


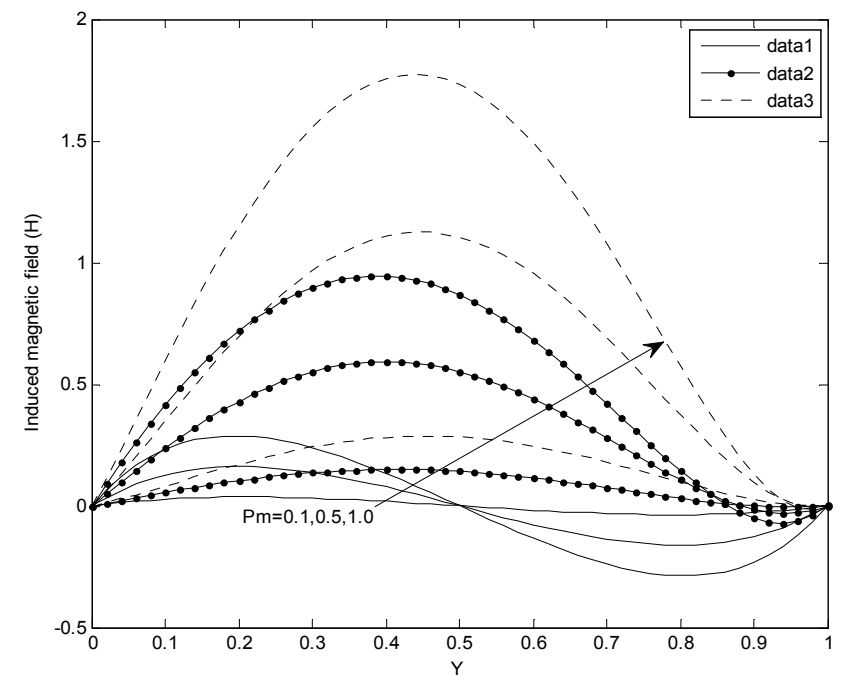

Fig.11. Variation of induced magnetic field with $\mathrm{Pm}(\mathrm{In}=1.667$, $\left.\mathrm{M}=5.0, \mathrm{Pm}=5, \mathrm{Gr} / \mathrm{Re}=100, \beta_{v} \mathrm{Kn}=0.05\right)$.

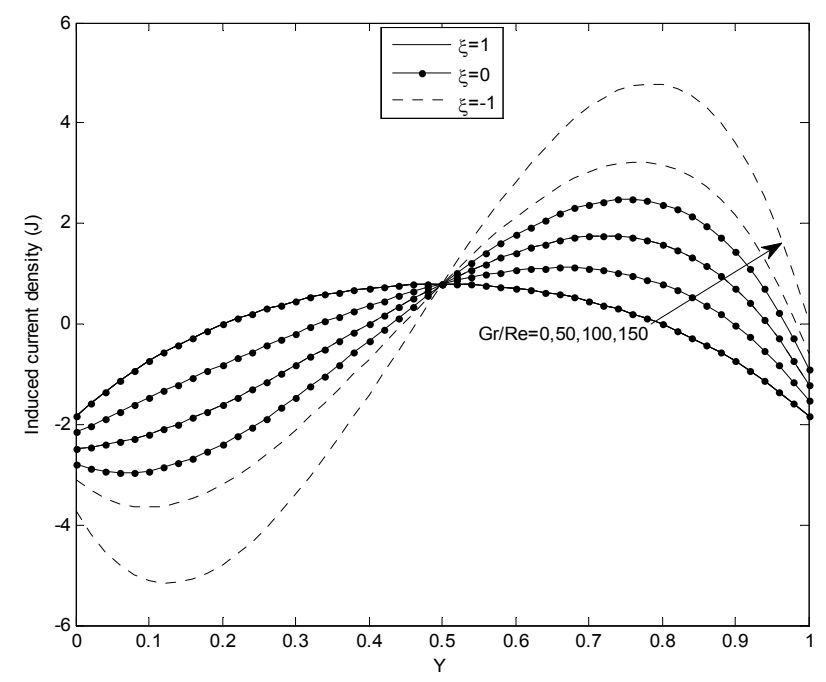

Fig. 12. Variation of induced current density with $\mathrm{Gr} / \mathrm{Re}$ $\left(\mathrm{In}=1.667, \mathrm{M}=5, \mathrm{Pm}=0.5, \beta_{v} \mathrm{Kn}=0.05\right)$.

It is evident from Fig.12 that increasing the mixed convection parameter ( $\mathrm{Gr} / \mathrm{Re})$ leads to a reduction in the fluid velocity at the channel wall $(y=0)$ while the result is just converse at the channel wall $(y=1)$. Further, at the middle of the channel, the induced current density is not affected by the mixed convection parameter $(\mathrm{Gr} / \mathrm{Re})$.

Figure 13 presents the variation of induced current density with respect to rarefaction parameter and wall-ambient temperature difference ratio. It is seen that an increase in the rarefaction parameter leads to an increase in the induced current density. It is also found that there exist points of intersection inside the microchannel where the induced current density is independent of the rarefaction parameter.

The variation of the induced current density with respect to fluid wall interaction parameter $(\ln )$ and wall-ambient temperature difference ratio $(\xi)$ is shown in Fig. 14. The induced current density decreases with the increase in the fluid wall interaction parameter in one part of the microchannel and a reverse trend occurs in the other part.

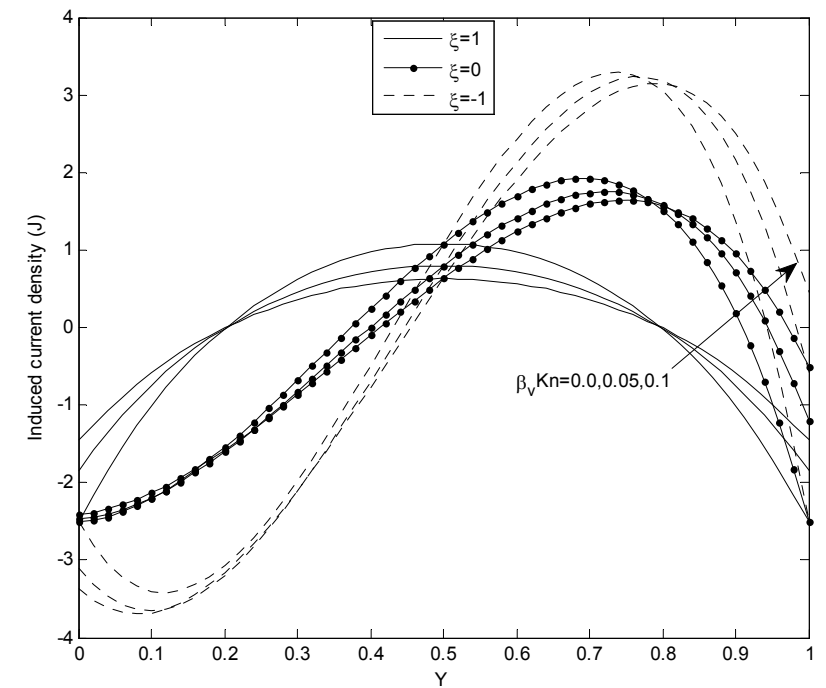

Fig.13. Variation of induced current density with $\beta_{v} \mathrm{Kn}$ $(\mathrm{In}=1.667, \mathrm{M}=5, \mathrm{Pm}=0.5, \mathrm{Gr} / \mathrm{Re}=100)$.

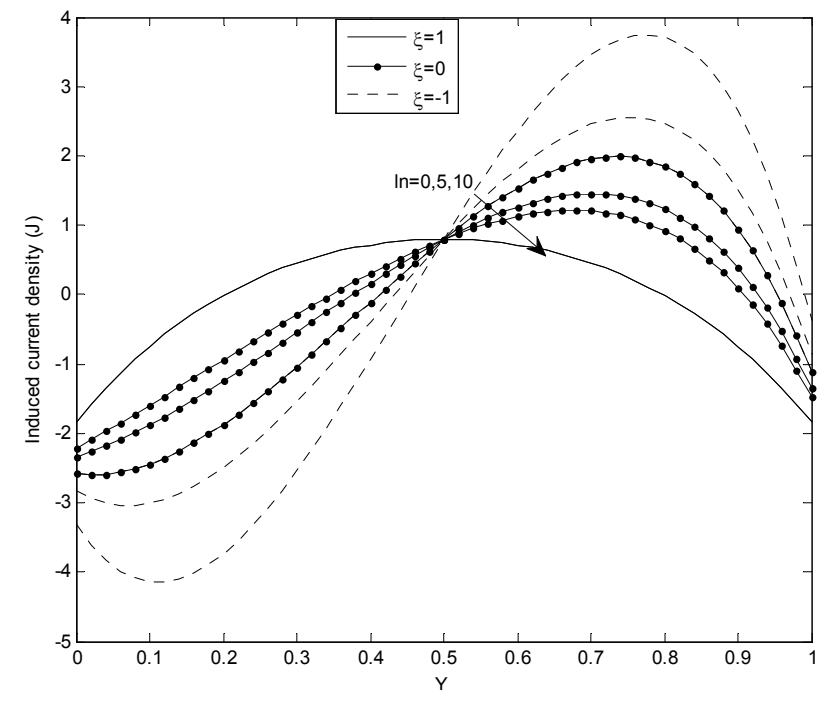

Fig.14. Variation of induced current density with In $\left(\beta_{v} \mathrm{Kn}=0.05, \mathrm{M}=5, \mathrm{Pm}=0.5, \mathrm{Gr} / \mathrm{Re}=100\right)$. 
Figures 15 and 16 exhibit the effects of the wall-ambient temperature difference ratio $(\xi)$, the Hartmann number $(\mathrm{M})$ and the magnetic Prandtl number $(\mathrm{Pm})$, respectively, on the induced current density for fixed values of $\beta_{v} K n=0.05, \ln =1.667$. It is evident from these figures that the effect of the Hartmann number and the magnetic Prandtl number on the induced current density is found to have a decreasing nature at the central region of the microchannel while a reverse trend occurs at the microchannel plates. Moreover, it is interesting to note that the current density changes its behaviour with the Hartmann number and the magnetic Prandtl number at two different locations inside the microchannel.

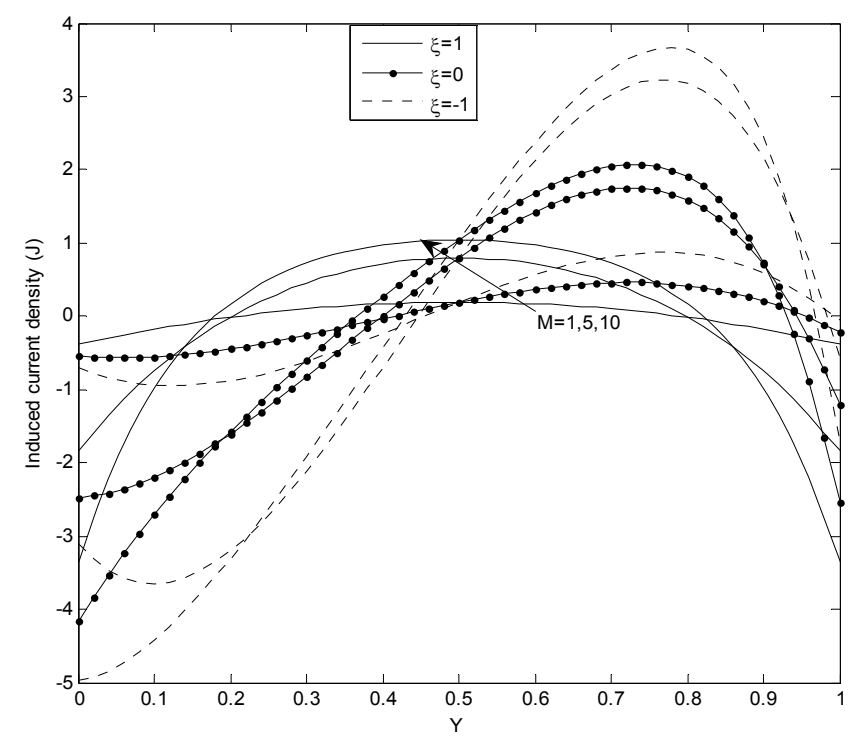

Fig.15. Variation of induced current density with $\mathrm{M}$ $\left(\beta_{v} \mathrm{Kn}=0.05, \mathrm{In}=1.667, \mathrm{Pm}=0.5, \mathrm{Gr} / \mathrm{Re}=100\right)$.

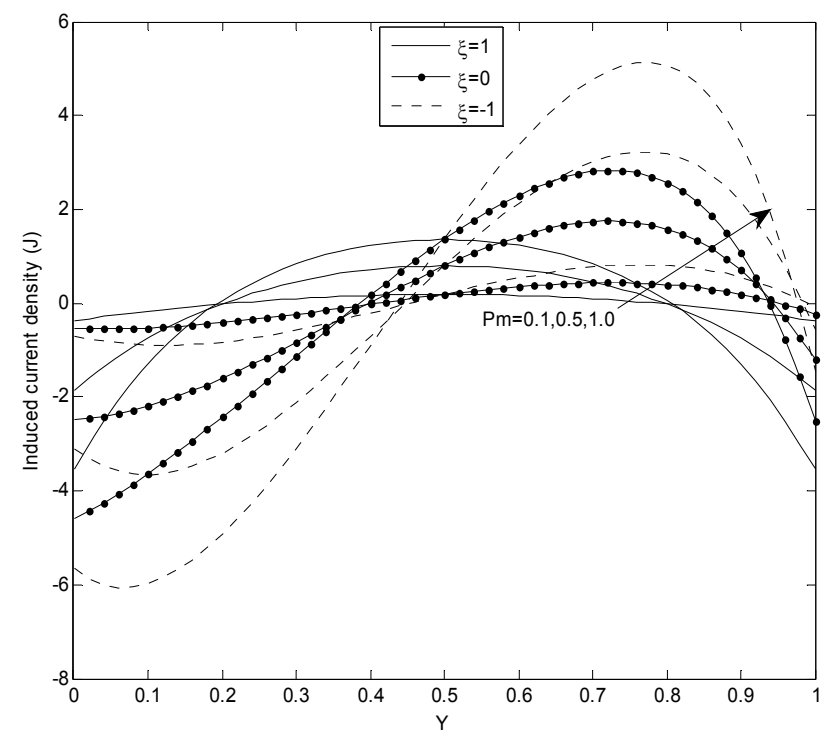

Fig.16. Variation of induced current density with Pm $\left(\beta_{v} \mathrm{Kn}=0.05, \mathrm{In}=1.667, \mathrm{M}=5, \mathrm{Gr} / \mathrm{Re}=100\right)$.

Figures 17 and 18 illustrate the effect of the rarefaction parameter and mixed convection parameter on the skin friction at the microchannel walls $y=0$ and $y=1$, respectively. It is observed that the skin friction decreases with an increase in the mixed convection parameter and rarefaction parameter at the microchannel wall $y=0$ while a reverse trend occurs at the microchannel wall $y=1$.

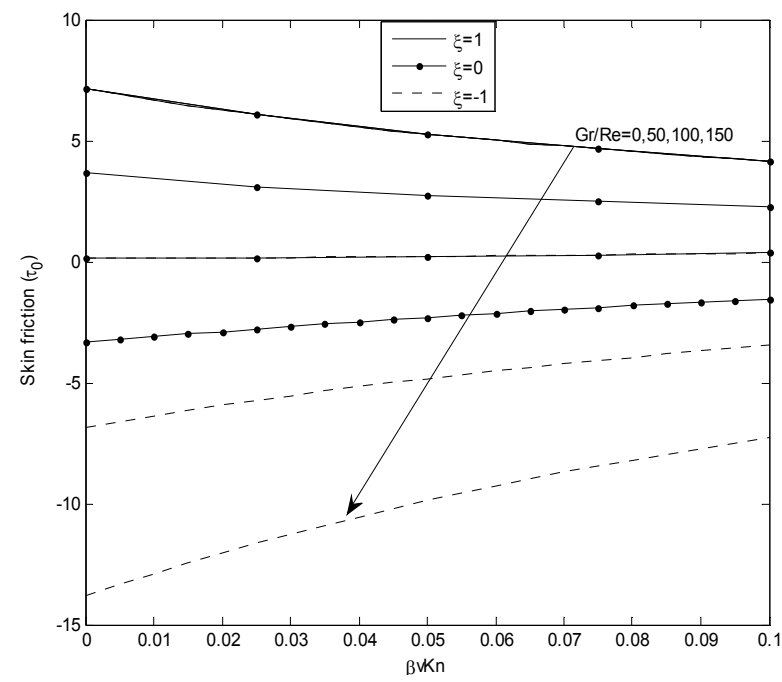

Fig.17. Variation of skin friction versus $\beta_{v} \mathrm{Kn}$ with $\operatorname{Gr} / \operatorname{Re}(Y=0)$.

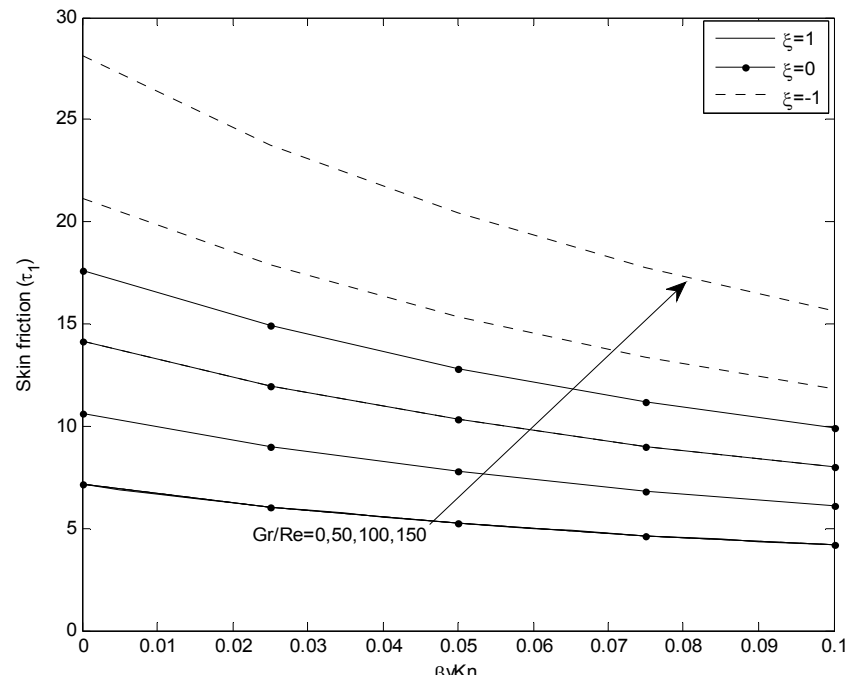

Fig.18. Variation of skin friction versus $\beta_{v} \mathrm{Kn}$ with $\mathrm{Gr} / \operatorname{Re}(Y=1)$. 
Figures 19 and 20 illustrate the effect of the fluid wall interaction parameter, rarefaction parameter, and wall-ambient temperature difference ratio on the skin friction. It is observed that the skin friction is enhanced with an increase in the fluid wall interaction at the microchannel wall $y=0$ while a reverse trend occurs at the microchannel wall $y=1$. Furthermore, it is found that the magnitude of skin friction is higher in the case of asymmetric heating in comparison with symmetric heating.

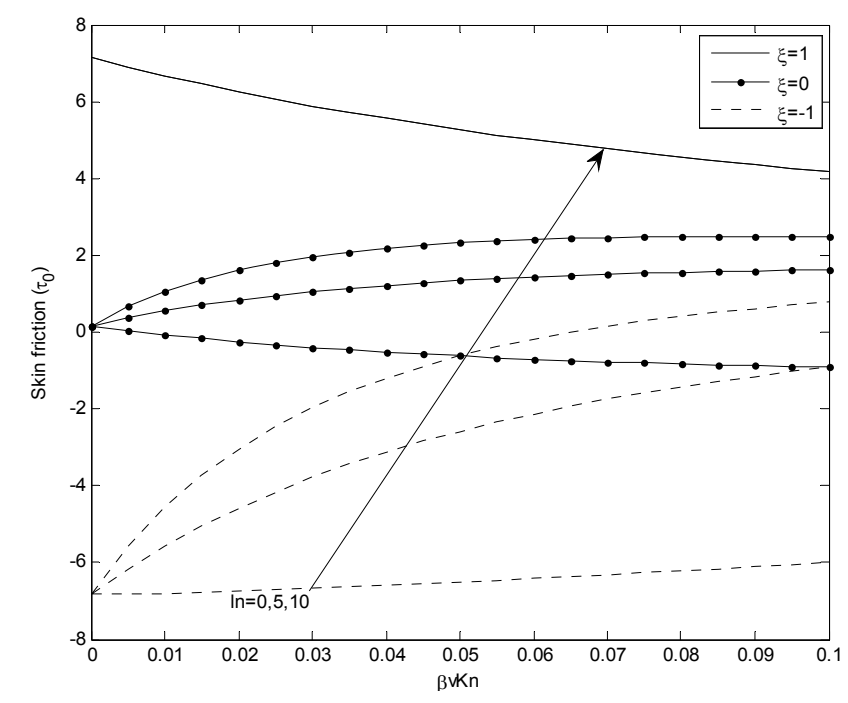

Fig.19. Variation of skin friction versus $\beta_{v} \mathrm{Kn}$ with In $(Y=0)$.

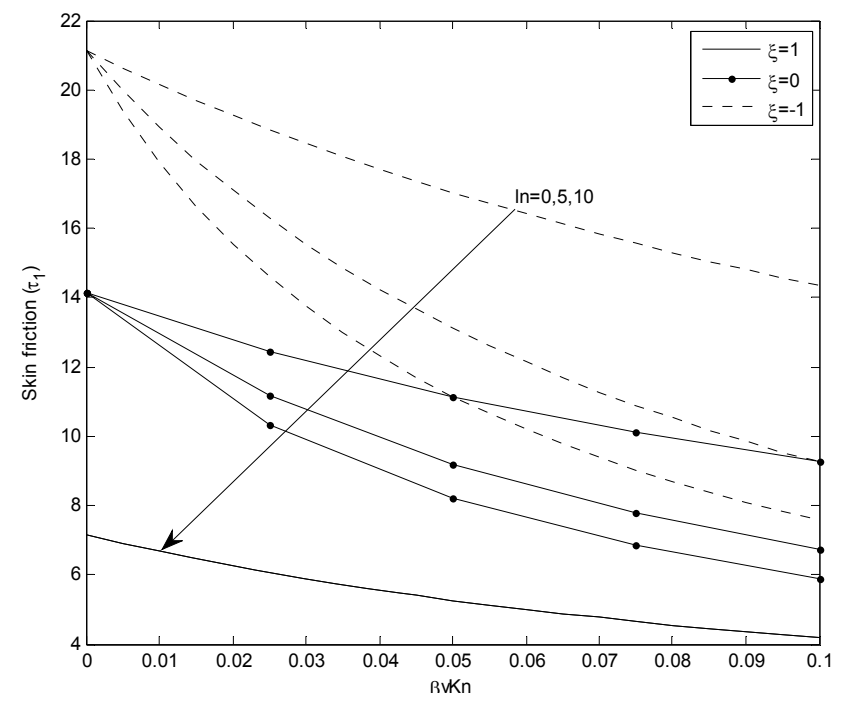

Fig.20. Variation of skin friction versus $\beta_{v} \mathrm{Kn}$ with In $(Y=1)$.

Figures 21, 22, 23 and 24 reveal the combined influences of the rarefaction parameter, the Hartmann number and the magnetic Prandtl number on the skin friction for different values of the wall-ambient temperature difference ratio at the microchannel walls $y=0$ and $y=1$, respectively. It is observed that an increase in the Hartmann number and the magnetic Prandtl number causes a reduction in the skin friction at the microchannel walls $y=0$ and $y=1$. It is further observed that the magnitude of the skin friction is higher in the case of asymmetric heating $(\xi=0,-1)$ in comparison with symmetric heating $(\xi=1)$.

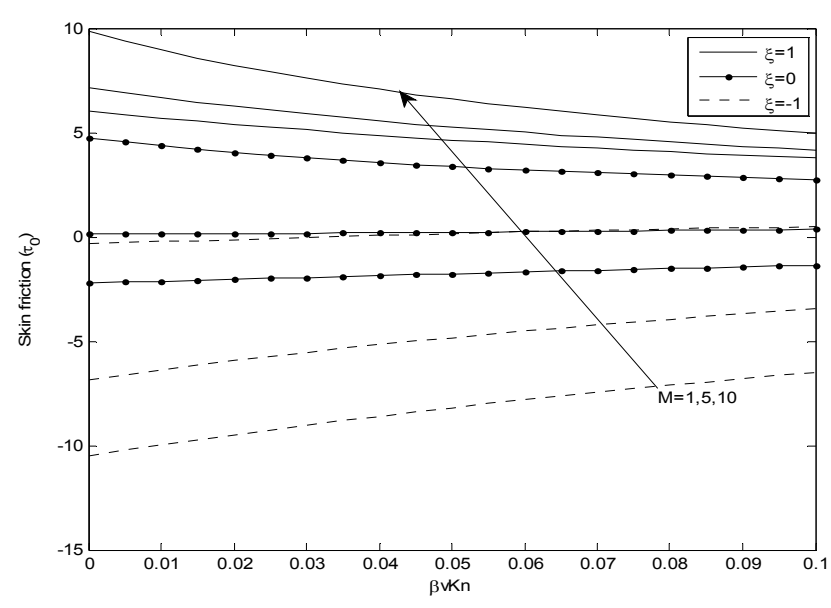

Fig.21. Variation of skin friction versus $\beta_{v} \mathrm{Kn}$ with $\mathrm{M}$ $(Y=0)$.

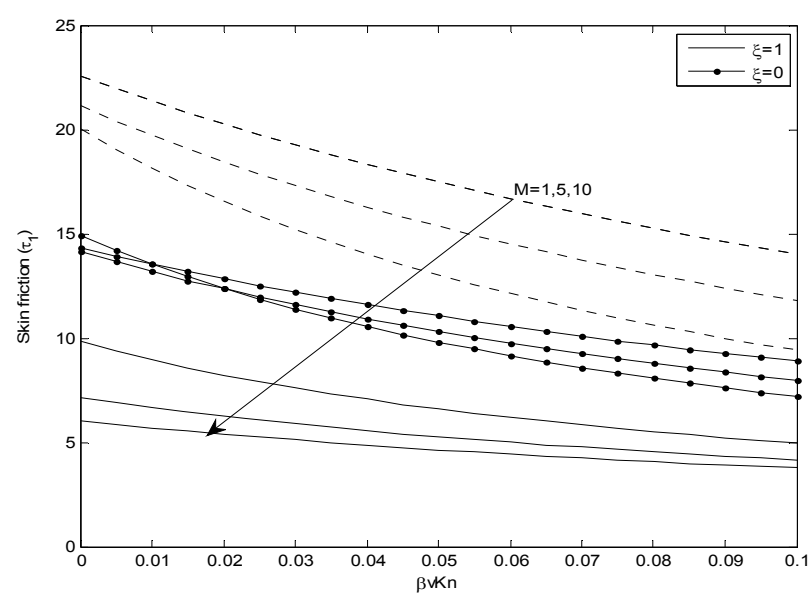

Fig.22. Variation of skin friction versus $\beta_{v} \mathrm{Kn}$ with $\mathrm{M}$ $(Y=1)$. 


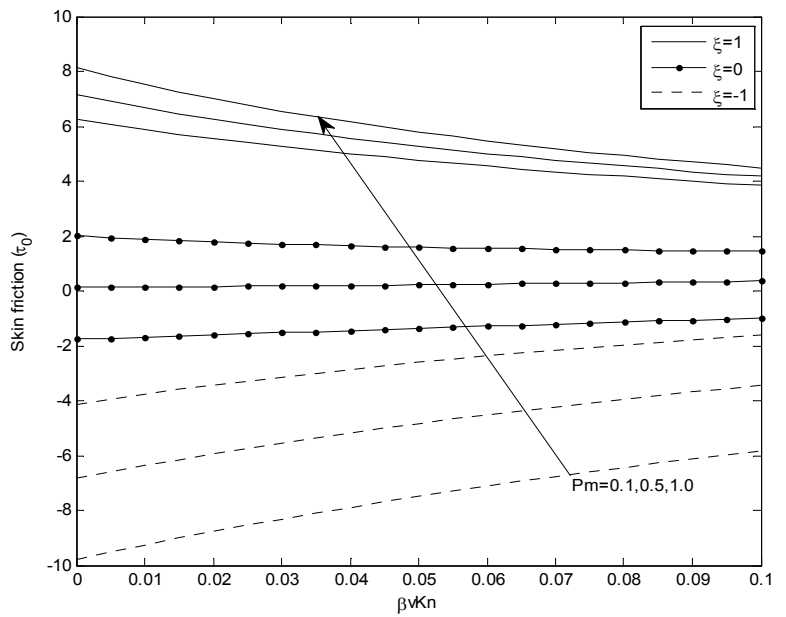

Fig.23. Variation of skin friction versus $\beta_{v} \mathrm{Kn}$ with $\mathrm{Pm}$ $(Y=0)$.

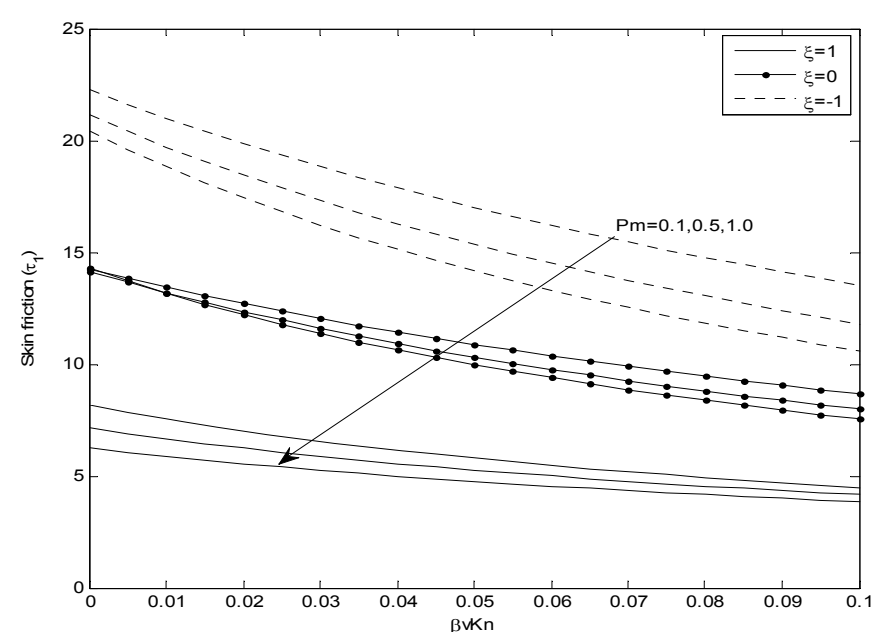

Fig.24. Variation of skin friction versus $\beta_{v} \mathrm{Kn}$ with $\mathrm{Pm}$ $(Y=1)$.

\section{Conclusions}

The effect of an induced magnetic field on the MHD mixed convection flow of a viscous, incompressible and electrically conducting fluid in the presence of a transverse magnetic field in a vertical microchannel formed by two electrically non-conducting infinite vertical parallel plates has been investigated analytically. The effects of various parameters on the velocity, induced magnetic field, temperature, induced current density and skin friction profiles have been shown in line graphs. The main findings are:

1. Increasing the value of the Hartmann number and the magnetic Prandtl number causes an enhancement in the induced magnetic field.

2. There exist points of intersection inside the microchannel where the induced magnetic field is independent of the Hartmann number and the magnetic Prandtl number and these strongly depend on the wall-ambient temperature difference ratio.

3. The effect of the Hartmann number and the magnetic Prandtl number on the induced current density is found to have a decreasing nature at the central region of the microchannel.

4. The magnitude of the skin friction is higher in the case of asymmetric heating $(\xi=0,-1)$ in comparison with symmetric heating $(\xi=1)$.

5. The effect of the Hartmann number on the skin friction can be useful in mechanical engineering for modelling a system. We can obtain a suitable value of the Hartmann number for which the value of the skin friction will be optimum.

6. This study exactly agrees with the findings of Chen and Weng [31] in the absence of a magnetic field.

\section{Appendix}

$$
\begin{aligned}
& C_{1}=\frac{(1-\xi)}{1+2 \beta_{v} \mathrm{Kn} \ln }, \quad C_{2}=\xi+\frac{\beta_{v} \mathrm{Kn} \ln (1-\xi)}{1+2 \beta_{v} \mathrm{Kn} \ln }, \quad C_{3}=A_{20}+\frac{d P}{d X} A_{21}-\frac{\mathrm{Gr}}{\mathrm{Re}} A_{22}, \\
& C_{5}=A_{25}+\frac{d P}{d X} A_{26}-\frac{\mathrm{Gr}}{\mathrm{Re}} A_{27}, \quad C_{4}=\beta_{v} \mathrm{Kn}\left[M \sqrt{\mathrm{Pm}} C_{5}+\frac{\mathrm{Gr}}{\mathrm{Re}} A_{1}\right]+A_{2} C_{3},
\end{aligned}
$$




$$
\begin{aligned}
& C_{6}=M \sqrt{\mathrm{Pm}} C_{5}+\frac{\mathrm{Gr}}{\mathrm{Re}} A, \quad A_{1}=\frac{C_{1}}{M^{2} \mathrm{Pm}}, \quad A_{2}=\frac{1}{M^{2} \mathrm{Pm}}, \\
& A_{3}=\cosh (M \sqrt{\mathrm{Pm}})+\beta_{v} \mathrm{Kn} M \sqrt{\mathrm{Pm}} \sinh (M \sqrt{\mathrm{Pm}}), \\
& A_{4}=\sinh (M \sqrt{\mathrm{Pm}})+\beta_{v} \operatorname{Kn} M \sqrt{\mathrm{Pm}} \cosh (M \sqrt{\mathrm{Pm}}), \\
& A_{5}=\frac{\mathrm{Gr}}{\mathrm{Re}}\left[-\beta_{v} \mathrm{Kn} A_{1}-A_{1}\right], \quad A_{6}=\frac{\mathrm{Gr}}{\mathrm{Re}} \beta_{v} \mathrm{Kn} A, \quad A_{7}=\beta_{v} \mathrm{Kn} M \sqrt{\mathrm{Pm}}, \quad A_{8}=-A_{3} A_{7}-A_{4}, \\
& A_{9}=A_{2}-A_{2} A_{3}, \quad A_{10}=A_{3} A_{6}-A_{5}, \quad A_{11}=A_{4}+A_{3} A_{7}, \quad A_{12}=-A_{2} A_{4}-A_{2} A_{7}, \\
& A_{13}=A_{4} A_{6}+A_{5} A_{7}, \quad A_{14}=\frac{A_{11}-A_{11} \cosh (M \sqrt{\mathrm{Pm}})}{\sinh (M \sqrt{\mathrm{Pm}})}, \quad A_{15}=\frac{A_{11}}{M \sqrt{\mathrm{Pm}} \sinh (M \sqrt{\mathrm{Pm}})}, \\
& A_{16}=0.5 C_{1}+C_{2}, \quad A_{17}=A_{16} A_{15}, \quad A_{18}=A_{9} A_{14}-A_{8} A_{12}, \quad A_{19}=A_{10} A_{14}-A_{8} A_{13}, \\
& A_{20}=\frac{A_{19}}{A_{18}}, \quad A_{21}=\frac{A_{15} A_{8}}{A_{18}}, \quad A_{22}=\frac{A_{17} A_{8}}{A_{18}}, \quad A_{23}=A_{8} A_{12}-A_{9} A_{14}, \\
& A_{24}=A_{10} A_{12}-A_{9} A_{13}, \quad A_{25}=\frac{A_{24}}{A_{23}}, \quad A_{26}=\frac{A_{15} A_{9}}{A_{23}}, \quad A_{27}=\frac{A_{17} A_{9}}{A_{23}}, \\
& A_{28}=\frac{\sinh (M \sqrt{\mathrm{Pm}})}{M \sqrt{\mathrm{Pm}}}, \quad A_{29}=\frac{\cosh (M \sqrt{\mathrm{Pm}})-1}{M \sqrt{\mathrm{Pm}}}, \quad A_{30}=\beta_{v} \mathrm{Kn} A_{28} M \sqrt{\mathrm{Pm}}, \\
& A_{31}=A_{28} A_{2}, \quad A_{32}=A_{26} A_{30}+A_{31} A_{21}+A_{29} A_{26}-A_{2} A_{21}, \\
& A_{33}=A_{27} A_{30}-\beta_{v} \mathrm{Kn} A_{1} A_{28}+A_{31} A_{22}+A_{29} A_{27}-A_{2} A_{22}-0.5 A_{1}, A_{34}=A_{2} A_{20}-A_{25} A_{29}-A_{25} A_{30}-A_{31} A_{20} .
\end{aligned}
$$

\section{Nomenclature}

\footnotetext{
$b$ - gap between the plates

$C_{\rho}$ - specific heat of the fluid at constant pressure

$g$ - gravitational acceleration

$H$ - dimensionless induced magnetic field

$H_{0}^{\prime} \quad$ - constant strength of the applied magnetic field

$H_{x}^{\prime} \quad$ - dimensional induced magnetic field

$J$-induced current density

Kn - Knudsen number

Ln - fluid wall interaction parameter
} 


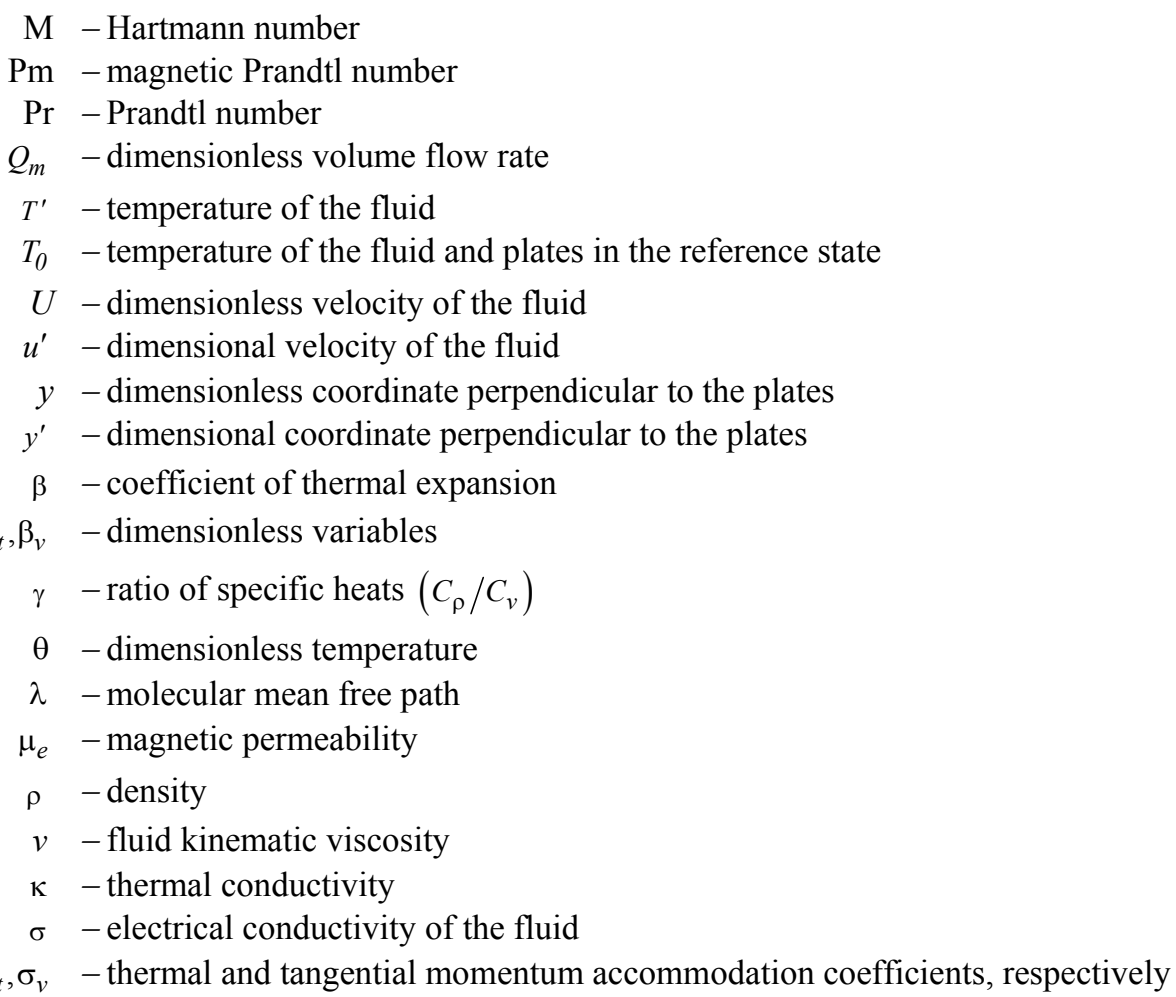

\section{References}

[1] Cramer K.R. and Pai S. (1973): Magneto fluid dynamics for engineers and applied physicists. - New York: McGraw-Hill, pp.204-237.

[2] Chawla S.S. (1967): Magnetohydrodynamics unsteady free convection. - ZAMM, vol.47, pp.499-508.

[3] Das S., Jana R.N. and Makinde O.D. (2014): MHD boundary layer slip flow and heat transfer of nanofluid past a vertical stretching sheet with non-uniform heat generation/absorption. - International Journal of Nanoscience, vol.13, No.3, 1450019.

[4] Sheikholeslamia M. and Gorji-Bandpy M. (2014): Free convection of ferro-fluid in a cavity heated from below in the presence of an external magnetic field. - Powder Technol.; vol.256, pp.490-8.

[5] Sheikholeslamia M., Gorji-Bandpy M., Ganji D.D., Rana P. and Soleimani Soheil (2014): Magnetohydrodynamic free convection of Al2O3-water nanofluid considering thermophoresis and Brownian motion effects. - Comput. Fluid, vol.94, pp.147-60.

[6] Sheikholeslamia M., Gorji-Bandpy M. and Ganji D.D. (2014): Lattice Boltzmann method for MHD natural convection heat transfer using nanofluid. - Powder Technol., vol.254, pp.82-93.

[7] Chaughan D. and Rastogi P. (2012): Heat transfer effects on a rotating MHD Couette flow in a Channel partially by a porous medium with Hall current. - Journal of Applied Science and Engineering, vol.15, No.3, pp.281-290.

[8] Ibrahim W. and Makinde O.D. (2015): Double-diffusive mixed convection and MHD stagnation point flow of nanofluid over a stretching sheet. - Journal of Nanofluids, vol.4, pp.28-37.

[9] Farhad A., Norzieha M., Sharidan S., Khan I. and Samiuthaq (2012): On hydromagnetic rotating flow in a porous medium with slip condition and Hall current. - International Journal of the Physical Sciences, vol.7, No.10, pp.1540-1548. 
[10] Farhad Ali, Ilyas Khan, Samiulhaq, Norzieha Mustapha and Sharidan Shafie (2012): Unsteady magnetohydrodynamic oscillatory flow of viscoelastic fluids in a porous Channel with heat and mass transfer.Journal of the Physical Society of Japan, vol.81, 064402.

[11] Jha B.K., Aina Babatunde and Ajiya A.T. (2015): MHD natural convection flow in a vertical parallel plate microchannel. - Ain Shams Engineering Journal, vol.6, pp.289-295.

[12] Jha B.K., Aina Babatunde and Ajiya A.T. (2015): Role of suction/injection on MHD natural convection flow in a vertical microchannel. - International Journal of Energy and Technology, vol.7, pp.30-39.

[13] Jha B.K., Aina Babatunde and Sani Isa (): Transient Magnetohydrodynamic Free Convective Flow in Vertical Micro-Concentric-Annuli. - Proc IMechE Part N: J Nanoengineering and Nanosystems, DOI: $10.1177 / 1740349915578956$

[14] Jha B.K., Aina Babatunde and Sani Isa (2015): Fully developed MHD natural convection flow in a vertical annular microchannel: an exact solution. - Journal of King Saud University- Science, vol.27, pp.253-259.

[15] Jha B.K. and Babatunde Aina (2016): MHD natural convection flow in a vertical micro-porous- annulus in the presence of radial magnetic field. - Journal of Nanofluids, doi:10.1166/jon.2016.1204

[16] Jha B.K., Babatunde Aina and Sani Isa (2015): MHD natural convection flow in a vertical micro-concentricannuli in the presence of radial magnetic field: an exact solution. - Journal of Ain Shams Engineering, http://dx.doi.org/10.1016/j.asej.2015.07.010

[17] Singh R.K., Singh A.K., Sacheti N.C. and Chandran P. (2010): On hydromagnetic free convection in the presence of induced magnetic field. - Heat Mass Transf., vol.46, pp.523-529.

[18] Jha B.K. and Sani I. (2013): Computational treatment of MHD of transient natural convection flow in a vertical Channel due to symmetric heating in presence of induced magnetic field. - J. Phys. Soc. Jpn, 82:084401.

[19] Ghosh S.K., Beg O.A. and Zueco J. (2010): Hydromagnetic free convection flow with induced magnetic field effects. - Meccanica, vol.14, pp.175-185.

[20] Kumar A. and Singh A.K. (2013): Unsteady MHD free convective flow past a semi-infinite vertical wall with induced magnetic field. - Appl Math. Comput., vol.222, pp.462-471.

[21] Sarveshanand and Singh A.K. (): Magnetohydrodynamic free convection between vertical parallel porous plates in the presence of induced magnetic field. - SpringerPlus, DOI 10.1186/s40064-015-1097-1

[22] Avci M. and Aydin O. (2007): Mixed convection in a vertical parallel plate microchannel. - ASME J. Heat Transfer, vol.129, pp.162-166.

[23] Eckert E.R.G. and Drake R.M. Jr. (1972): Analysis of heat and mass transfer. - New York: McGraw-Hill, Chap. 11.

[24] Goniak R. and Duffa G. (1995): Corrective term in wall slip equations for Knudsen layer. - J. Thermophys. Heat Transfer, vol.9, pp.383-384.

[25] Chen C.K. and Weng H.C. (2005): Natural convection in a vertical microchannel. - J. Heat Transfer, vol.127, pp.1053-1056.

Received: June 18, 2016

Revised: June 11, 2017 